\title{
Data Mining Approach for Educational Decision Support
}

\author{
Sinta Septi Pangastuti ${ }^{1{ }^{*}}$, Kartika Fithriasari ${ }^{2}$, Nur Iriawan $^{3}$, Wahyuni Suryaningtyas ${ }^{4}$ \\ 1 Statistics Department, Universitas Padjadjaran \\ 2,3 Statistics Department, Institut Teknologi Sepuluh Nopember \\ 4 Mathematics Education, Muhammadiyah University of Surabaya \\ * Corresponding author: sinta.septi@unpad.ac.id
}

Received: 1 March 2020 ; Accepted: 18 January 2021 ; Published: 21 January 2021

\begin{abstract}
: data mining techniques in education sector have begun to evolve, along with the development of technology and the amount of data that can be stored in an education database storage system. One of them is a database of Bidikmisi scholarships in Indonesia. The Bidikmisi data used in this study will be classified using classification data mining technique. The technique that used in this study is random forest in combination with boosting algorithm and bagging algorithms. These algorithms also combine with SMOTE algorithm to handling the imbalance class in dataset. Based on the performance criteria G-mean and AUC, the algorithm combines with SMOTE tended to be better. The classification accuracy of each method being more than $90 \%$.
\end{abstract}

Keywords: Bidikmisi, Bagging, Boosting, Classification, Educational data mining

\section{Introduction}

Research on machine learning has produced techniques for data mining that are able to find out the hidden relationships between variables or between indicators. Data mining has become a very useful tool for extracting and manipulating data, and making patterns in large amounts of data, so as to produce information that is useful for decision making. In this research, we will discuss Educational Data Mining (EDM), defined as the area of scientific inquiry centered around the development of methods for making discoveries within the unique kinds of data that come from educational settings [1]. Prediction methods are used in Bidikmisi scholarship data to obtain additional information regarding scholarship recipients. The process of determining is not easy, due to considering a large number of prospective applicants and variable criteria. In the case of prediction models, the label for the output variable is known in advance, namely the status of scholarship acceptance (binary type, 0 and 1 ).

This study aims to use data mining classification analysis. Previous research has been conducted by paying attention to imbalances in their prediction class, using boosting to deal with the problem of classification imbalance [2]. Furthermore, researchers are interested in comparing the results of boosting and bagging methods. The final result is obtained a predictive value from the method of boosting and bagging to represent the conditions of receiving scholarships in Indonesia.

\section{Problem Description}

The data selected for this research is the educational dataset, which is Bidikmisi scholarship data. The goal is to provide opportunities for people with medium to low economic conditions. However, there are indications of problems with the implementation of the Bidikmisi scholarship program, i.e. the existence of unacceptable admission conditions. The main factors for scholarship holders in Bidikmisi are Parent Revenue and the Total Person per Household [3]. According to Suryaningtyas et al. [4] the condition is identified as the category of acceptance with the "true" conditions and the acceptance conditions of the "false" category. The condition is considered as "true" if students are accepted Bidikmisi with conditions of incapacity and vice versa. Furthermore, acceptance is categorized as "false" if the student is accepted 
Bidikmisi with capable conditions and vice versa. For the above reasons, the researchers chose to use the boosting and bagging algorithm to classify the Bidikmisi imbalance data. Two boosting algorithms are used, namely AdaBoost and SMOTE boosting, and one method, SMOTE bagging. Previously, random forest classification parameter optimization was used, which was then used as the base classifier for boosting and bagging models.

Table 1. Criteria of Bidikmisi Scholarship

\begin{tabular}{|c|c|c|c|c|}
\hline $\mathbf{Y}$ & $\mathrm{CC}$ & $\mathrm{AC}$ & Criteria & Interpretation \\
\hline 1 & 0 & 0 & False & $\begin{array}{l}\text { Acceptance criteria is false if the grantee }(Y=1) \text { is followed with the } \\
\text { category of a wealthy family }\end{array}$ \\
\hline 0 & 1 & 0 & False & $\begin{array}{l}\text { Acceptance criteria is false if the grantee }(Y=0) \text { is followed with the } \\
\text { category of a poor family }\end{array}$ \\
\hline 1 & 1 & 1 & True & $\begin{array}{l}\text { Acceptance criteria is true if the grantee }(Y=1) \text { is followed with the } \\
\text { category of a poor family }\end{array}$ \\
\hline 0 & 0 & 1 & True & $\begin{array}{l}\text { Acceptance criteria is true if the grantee }(Y=0) \text { is followed with the } \\
\text { category of a wealthy family }\end{array}$ \\
\hline
\end{tabular}

Source: Suryaningtyas (2010)

\section{Literature Review}

\section{Boosting Algorithm}

Boosting is one of the ensemble methods that improve the performance of a learning algorithm by combining a collection of weak classifiers into a strong final classifier. The main idea of the boosting process is to select a set of training data (training examples) in a number of ways, which are then examined by a basic learner. This process can be accomplished by selecting training patterns that are expected to make the performance of the base classifier worse than even the performance of the base classifier on a regular basis. If this can be achieved, it is hoped that the base learner can create a new base classifier in the next iteration that is significantly different from its predecessor. This is because the base learner is expected to be a weak algorithm, but may also provide a base learner with the output of a classifier that does not make clear predictions [5]. The boosting algorithm used in this study is Adaptive Boosting M2, an extension algorithm from the original boosting algorithm.

Adaptive boosting M2 algorithm:

$$
\begin{aligned}
& \text { Input: }\left(\mathbf{x}_{i}, y_{i}\right), \ldots,\left(\mathbf{x}_{m}, y_{m}\right) \text { where } \mathbf{x}_{i} \in \mathbf{X} \text { dan } y_{i} \in \mathbf{Y}=\{1, \ldots, k\} \\
& \text { Given: } B=\left\{(i, y): i \in\{1, \ldots, m\}, y \neq y_{i}\right\} \\
& \text { Initial: } D_{1}(i, y)=1 /|B| \text { for }(i, y) \in B \\
& \text { for } t=1,2, \ldots, T
\end{aligned}
$$

Train weal learner use the distribution of $D_{t}$

Compute a weak hypothesis $h_{t}: X \times \mathrm{Y} \rightarrow[0,1]$ with pseudo-loss, Equation (1).

$$
\varepsilon_{t}=\frac{1}{2} \sum_{(i, y) \in B} D_{t}(i, y)\left(1-h_{t}\left(x_{i}, y_{i}\right)+h_{t}\left(x_{i}, y_{i}\right)\right)
$$

if $e_{t}>0,5$, then the learning process stops.

Set $\beta_{t}=\frac{\varepsilon_{t}}{\left(1-\varepsilon_{t}\right)}$

Update weight values

$D_{t+1}(i, y)=\frac{D_{t}(i, y)}{Z_{t}} \times \beta_{t}^{\left(\frac{1}{2}\right)\left(1+h_{t}\left(x_{i}, y_{i}\right)-h_{t}\left(x_{i}, y_{i}\right)\right)}$ 
where $Z_{t}$ is a normalization contact that makes $\sum_{i=1}^{m} D_{t+1}(i, y)=1$

Output:

$$
H(x)=\arg \max _{y \in Y} \sum_{t=1}^{T}\left(\log \frac{1}{\beta_{t}}\right) h_{t}(x, y)
$$

\section{Bagging Algorithm}

Bagging is a method of modifying the results of classification algorithms in machine learning. This method was introduced by [6] and stands for the word "bootstrap aggregating". In the classification with two possible classes, the classification algorithm forms the $\mathrm{H}$ : D classifier $\rightarrow\{-1,1\}$ as the basis for the training data. The bagging method determines the order of the $H t$ classifier, where $\mathrm{t}=1, \ldots, \mathrm{T}$ as a modification of the training data. The classifier is then combined into a combined classifier. The prediction results of the combined classifier are then given as combined weights of the individual classifier or referred to as the voting procedure. According to [6], bagging is an effective ensemble technique for unstable learning algorithms, where small changes in training data sets can make large changes in predictions for e.g. decision trees, neural networks, etc.

\section{Synthetic minority Over-Sampling (SMOTE) Algorithm}

SMOTE is one of the Chawla [7] methods that can handle the imbalanced dataset. The basic idea of SMOTE is to increase the number of samples in the minor class. It allows to match the main class by generating synthetic data based on the $\mathrm{k}$ nearest neighbor. In this case the nearest neighbor based on the Euclidean distance between data. Given a dataset with $p$ variables $\mathbf{x}^{T}=\left\lfloor\mathbf{x}_{1}, \mathbf{x}_{2}, \ldots, \mathbf{x}_{p}\right\rfloor$ and $\mathbf{z}^{T}=\left[\mathrm{z}_{1}, \mathrm{z}_{2}, \ldots, \mathrm{z}_{p}\right]$ then the Euclidean distance $d(x, z)$ generally as below:

$$
d(\mathbf{x}, \mathbf{z})=\sqrt{\left(\mathrm{x}_{1}-\mathrm{z}_{1}\right)^{2}+\left(\mathrm{x}_{2}-\mathrm{z}_{2}\right)^{2}+\ldots+\left(\mathrm{x}_{p}-\mathrm{z}_{p}\right)^{2}}
$$

Generate synthetic data using the following equation:

$$
\mathbf{x}_{s y n}=\mathbf{x}_{i}+\left(\mathbf{x}_{k n n}-\mathbf{x}_{i}\right) \gamma
$$

where:

$\mathrm{X}_{\text {syn: synthetic data }}$

$\mathrm{x}_{\mathrm{i}}$ : the $i$-th data from the minor class

$\mathrm{x}_{\mathrm{knn}}$ : data from the minor class having the smallest distance to $\mathrm{x}_{\mathrm{i}}$

$\gamma$ : a random number between 0 and 1 .

\section{SMOTE-Boosting Algorithm}

This algorithm was proposed by [7] in 2002. SMOTE-boosting combines the SMOTE algorithm and the standard boosting procedure, by utilizing SMOTE to improve the prediction of minority classes. The purpose of combining the SMOTE and AdaBoost algorithms is to increase the value of the True Positive (TP) rate [7]. In the AdaBoost iteration procedure, the classifier component classification results are first put into probabilities form, which is later used to calculate the pseudo-loss.

SMOTE-boosting algorithm:

Input: $\left(\mathbf{x}_{i}, y_{i}\right), \ldots,\left(\mathbf{x}_{m}, y_{m}\right)$ where $\mathbf{x}_{i} \in \mathbf{X}$ dan $y_{i} \in \mathbf{Y}=\{1, \ldots, k\}$

Given: $B=\left\{(i, y): i \in\{1, \ldots, m\}, y \neq y_{i}\right\}$

Initial: $D_{1}(i, y)=\frac{1}{|B|}$ for $(i, y) \in B$

for $\mathrm{t}=1, \ldots, \mathrm{T}$

Modify the distribution of $D_{t}$ by creating $\mathrm{N}$ synthetic examples from minority class using the

SMOTE algorithm.

Train weak learner using the distribution of $D_{t}$

Compute a weak hypothesis with pseudo-loss in Equation (7). 


$$
\varepsilon_{t}=\sum_{(i, y) \in B} D_{t}(i, y)\left(1-h_{t}\left(x_{i}, y_{i}\right)+h_{t}\left(x_{i}, y_{i}\right)\right)
$$

if $e_{t}>0,5$, then the learning process stops.

Set $\beta_{t}=\frac{\varepsilon_{t}}{\left(1-\varepsilon_{t}\right)}$

Update weight value:

$$
D_{t+1}(i, y)=\frac{D_{t}(i, y)}{Z_{t}} \times \beta_{t}^{\left(\frac{1}{2}\left(1+h_{t}\left(x_{i}, y_{i}\right)-h_{t}\left(x_{i}, y_{i}\right)\right)\right.}
$$

where $Z_{t}$ is a normalization contact that makes $D_{t+1}(i, y)=1$

Output:

$$
H(x)=\arg \max _{y \in Y} \sum_{t=1}^{T}\left(\log \frac{1}{\beta_{t}}\right) h_{t}(x, y)
$$

\section{SMOTE-Bagging Algorithm}

SMOTE-bagging algorithms is a combination of SMOTE as mention earlier and bagging algorithms. SMOTE-bagging involves steps to generate synthetic data while creating subsets [8]. Based on SMOTEbagging, each subset obtained by the bootstrap process is matched with SMOTE before the model is formed. Synthetic data is created based on two parameters, the amount of oversampling $(\mathrm{N})$ from the minority class and $\mathrm{k}$ closest neighbors. Total oversampling is decided so that the number of major and minor classes is balanced.

SMOTE-bagging algorithm:

Let $D$ the training dataset

for $t=1,2, \ldots, T$, construct subset $D t$ from classes with the same number by executing the following:

Resample class $C$ with replacement at $100 \%$

For each class $i(1,2, \ldots, C-1)$ :

Resample from original instances with replacement, where $b \%$ is multiple of $100 \%$

Set $N=\left(N_{C} / N_{i}\right) \cdot(1-b \%) \cdot 100$

Generate new instances by using $\operatorname{SMOTE}(k, N)$

Train a classifier from $D_{t}$

Change percentage $b \%$

Repeat step 2 and 3 until convergent

Testing on a new instance:

Generate outputs from each classifier

Return the class which gets the most votes from classifier $H t: D t \rightarrow R$

$$
H\left(d_{i}, c_{j}\right)=\operatorname{sign}\left(\sum_{t=1}^{T} \alpha_{t} H_{t}\left(d_{i}, c_{j}\right)\right)
$$

\section{Performance Criteria for Classification}

Confusion matrix contains information about the actual data class represented on the matrix row and the predictive data class on the column [9], it has actual and predictive data from the classification model, then presented using a cross-tabulation. 
Table 2. Confussion Matrix

\begin{tabular}{lccc}
\hline & & \multicolumn{2}{c}{ Predictive } \\
& & Positive Class & Negative Class \\
\hline \multirow{2}{*}{ Real } & Positive Class & True Positive (TP) & False Negative (FN) \\
& Negative Class & False Positive (FP) & True Negative (TN) \\
\hline
\end{tabular}

Area Under Curve (AUC) have been used to understand the performance of learning algorithms in minority classes. Then, to evaluate the overall method's performance, a geometric mean (G-mean) is employed. G-mean is a geometric mean of sensitivity and specificity. If all positive classes cannot be predicted, the G-mean will be zero so that a classification algorithm is expected to reach a high G-mean value [10]. Therefore, in this paper, to evaluate the overall method performance, a G-mean and AUC analysis can be used.

$$
\begin{aligned}
& \text { Specificity }=\frac{\mathrm{TN}}{(\mathrm{TN}+\mathrm{FP})} \times 100 \% \\
& \text { Sensitivity }=\frac{\mathrm{TP}}{(\mathrm{TP}+\mathrm{FN})} \times 100 \% \\
& G-\text { Mean }=\sqrt{\text { Sensitivity } \times \text { Specificity }}
\end{aligned}
$$

AUC obtained by calculating the value of true positive rate, which is the number of objects in positive classes that are correctly classified (TPR) and false positive rate, which is the number of objects in positive classes that are incorrectly classified (FPR).

$$
\begin{aligned}
& \mathrm{TPR}=\frac{\mathrm{TP}}{(\mathrm{TP}+\mathrm{FN})} \\
& \mathrm{FPR}=1-\frac{\mathrm{TN}}{(\mathrm{TN}+\mathrm{FP})} \\
& \mathrm{AUC}=\frac{1+\mathrm{TPR}-\mathrm{FPR}}{2}
\end{aligned}
$$

\section{Methodology}

\section{Source of Dataset}

The data used is the Database of the Ministry of Research, Technology, and Higher Education through Bidikmisi channel. We used dataset from East Java province in 2013 - 2017.

Table 3. Data Description

\begin{tabular}{ccccc}
\hline Data & $\begin{array}{c}\text { Total Data } \\
(\mathbf{N})\end{array}$ & Variable & \multicolumn{1}{c}{ Categories (Y) } \\
\hline Bidikmisi & 52631 & 11 & 1 & $\begin{array}{l}\text { Accepted }=50796 \\
\text { Unaccepted }=\end{array}$ \\
\hline
\end{tabular}

\section{Reserch Design}

In this research study, the experiments were used to perform on the Bidikmisi dataset summarize in Table 2, the dataset contains 52631 records and 11 variables. The variables can be classified as demographic attributes (such as occupation, education, housing ownership, area of residential land, area of a residential building, etc). The number of categories distributed into type, as we can see in Fig. 1. Classification analysis procedure using boosting and bagging algorithms is given in the research flowchart, as seen in Fig. 2. 


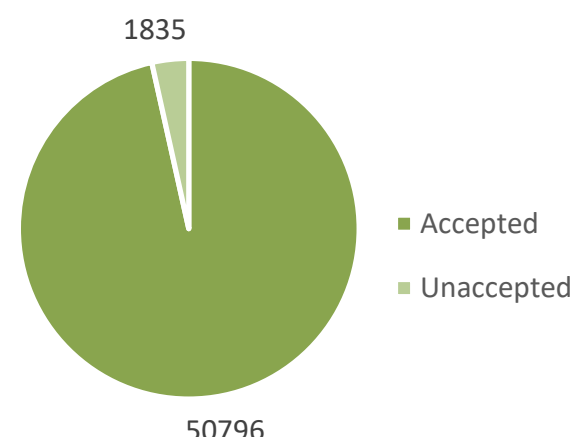

Figure 1. Data Characteristic Based on Student Status

\section{Tools for Analysis}

As we mentioned before, this study aims to determine which classification analysis had the best performance. By competing the ensemble methods which are AdaBoost, SMOTE-Boosting, SMOTEBagging. The methods is analyzed using $\mathrm{R}$ software and ebmc package.

\section{Result and Discussion}

\section{Preprocessing Bidikmisi Dataset}

The steps involved in preprocessing data include combining data from various sources, cleaning data to remove noise, filling missing value and eliminating outliers. In this paper, we cleaned the missing value, therefore, there is no noise in the dataset. From Table 4, there was a missing value for each variable that will be removed from the study. Therefore, the total data used for research is 52631, after detecting the missing value and handling it by deleting the data.

Table 4. Missing Value in Bidikmisi Dataset

\begin{tabular}{cccc}
\hline & \multicolumn{2}{c}{$\mathrm{N}$} & $\begin{array}{c}\% \\
\text { Missing }\end{array}$ \\
\cline { 2 - 3 } X1 & Valid & Missing & 0 \\
\hline X2 & 53661 & 0 & 0 \\
\hline X3 & 53661 & 0 & 0 \\
\hline X4 & 53661 & 0 & 0 \\
\hline X5 & 53661 & 0 & 0 \\
\hline X6 & 52861 & 0 & 1,5 \\
\hline X7 & 52884 & 777 & 1,4 \\
\hline X8 & 53661 & 0 & 0 \\
\hline X9 & 53661 & 0 & 0 \\
\hline X10 & 53130 & 531 & 1 \\
\hline X11 & 53190 & 471 & 0,9 \\
\hline
\end{tabular}




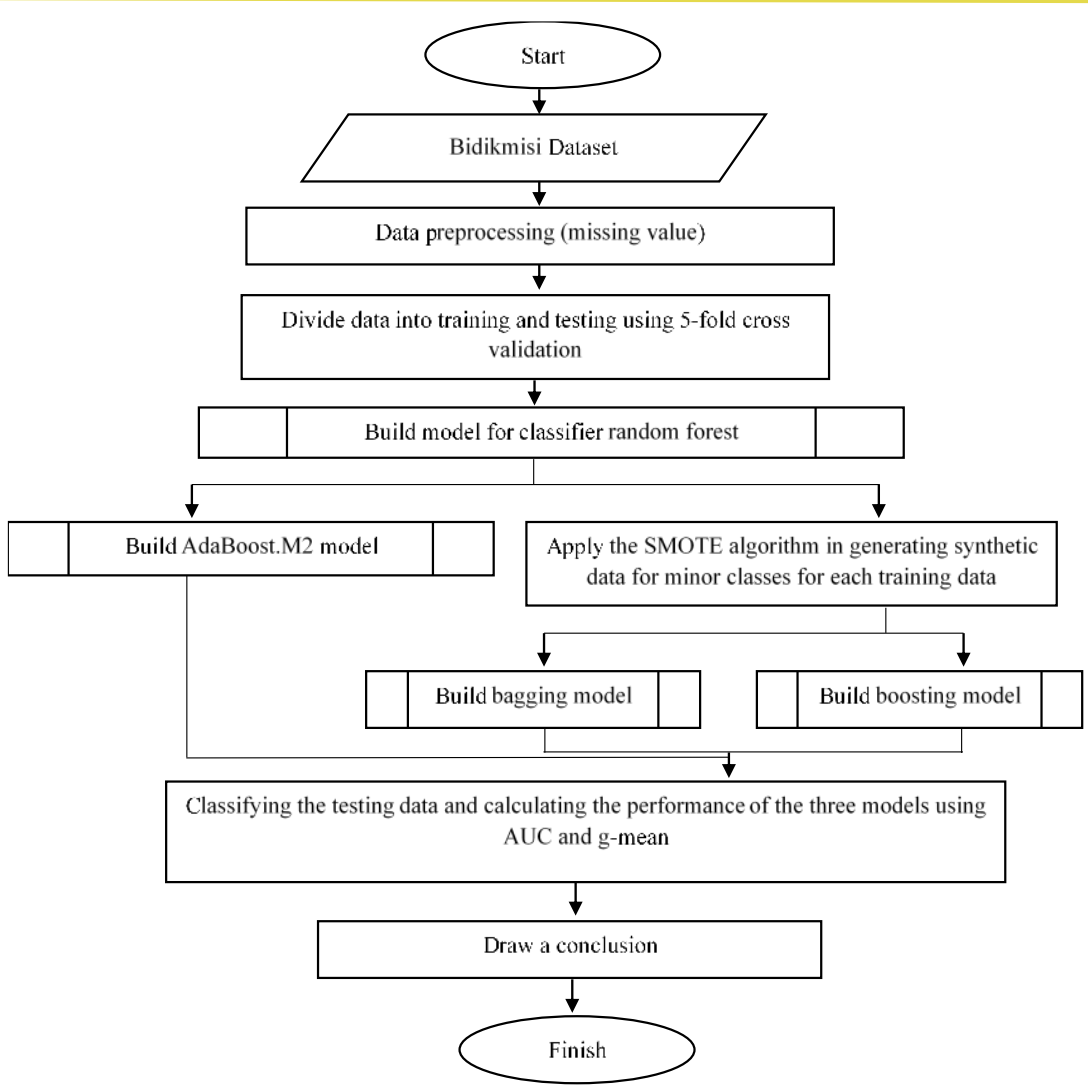

Figure 2. Flowchart Analysis on bidikmisi Dataset

\section{Adaptive Boosting Classification Analysis}

By dividing the dataset into training data and testing data, the model for AdaBoost was formed. This study used 5 -fold cross-validation with a $20 \%$ partition for each fold. Fig. 3 shown the performance criteria G-means and AUC for adaptive boosting algorithm, the value G-mean and AUC tend to reach a peak when the iteration is 50 , with $50.256 \%$ and $9.027 \%$.

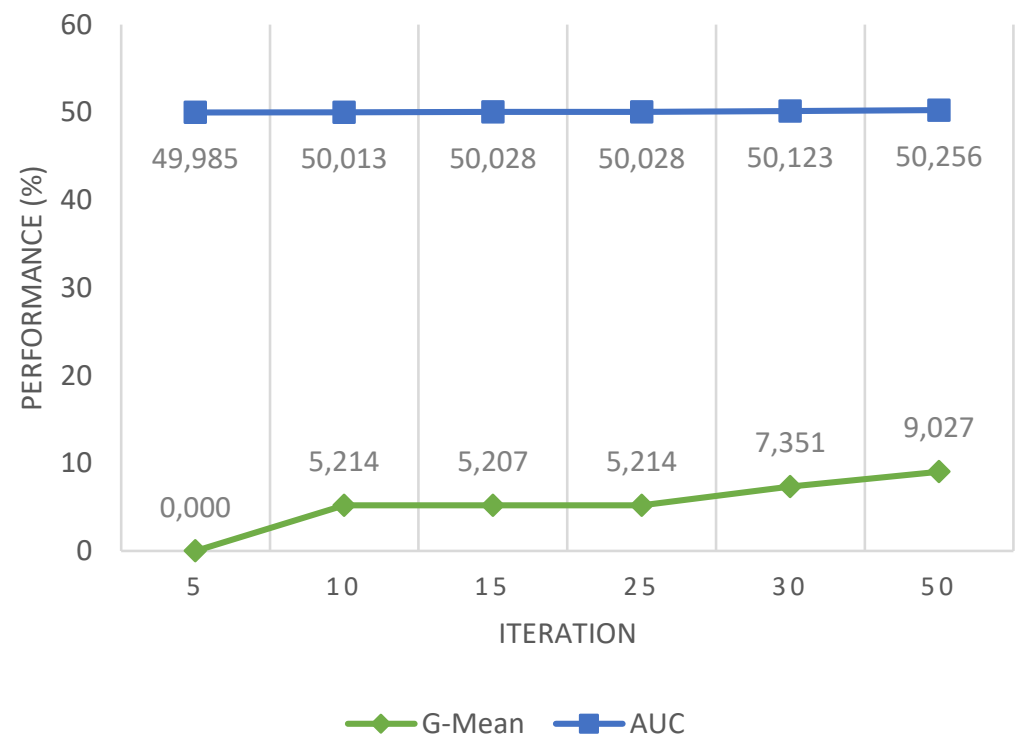

Figure 3. G-mean and AUC Value for Adaptive Boosting Algorithm 


\section{SMOTE_Boosting Classification Analysis}

As we discussed before, the dataset divided into two sets of training data and testing data using 5-fold crossvalidation. Then, create synthetic data to balance the class composition and minor classes using the SMOTE algorithm. Therefore, we conclude the G-mean and AUC values as shown in Fig. 4.

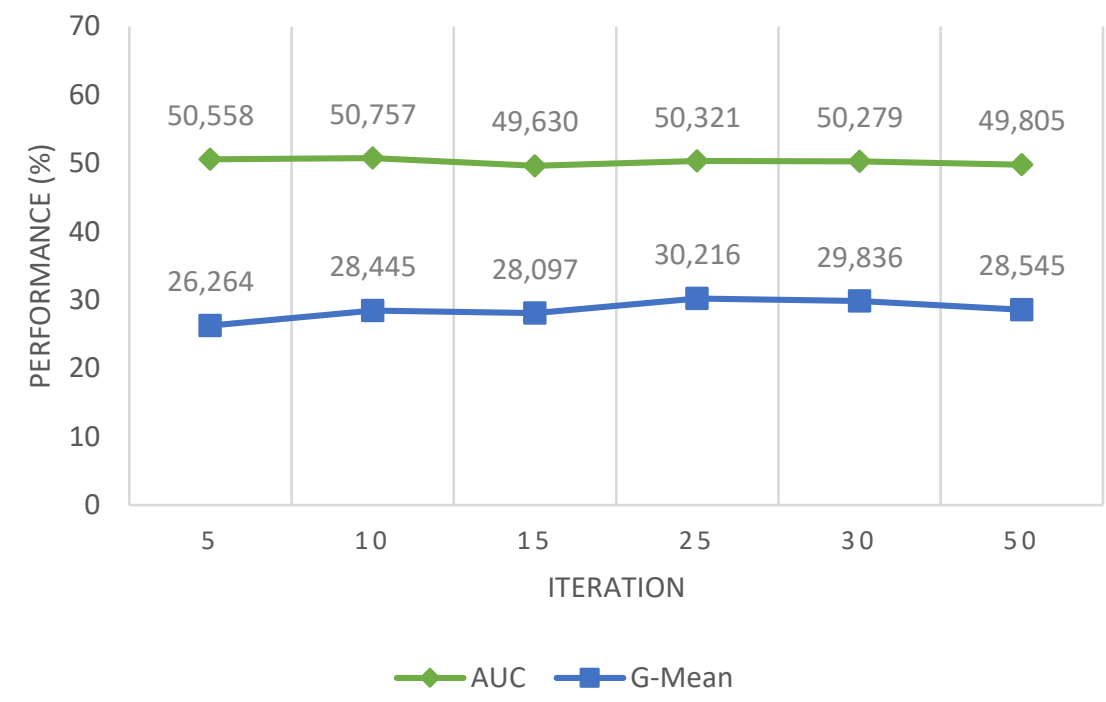

Figure 4. G-mean and AUC Value for SMOTE-Boosting Algorithm

Fig. 4 shows the method performance, G-mean and AUC values tend to be stable without a drastic increase. This value is bigger than the previous methods.

\section{SMOTE-Bagging Classification Analysis}

The initial step that must be done in the analysis of the bagging method has divided the data into training and testing dataset with 5-fold cross-validation. Then, bootstrapping is performed on the training data. Because this method combines bagging and SMOTE algorithm, after bootstrapping the data will be generated again using SMOTE. Fig. 5 shows the classification performance for the Bidikmisi dataset using 5 iterations. The G-mean value reached a maximum at the $10^{\text {th }}$ iteration, with a value of $33.129 \%$ and the maximum AUC value at the $50^{\text {th }}$ iteration was $32.924 \%$. This method slightly better than the previous methods based on the G-mean and AUC values.

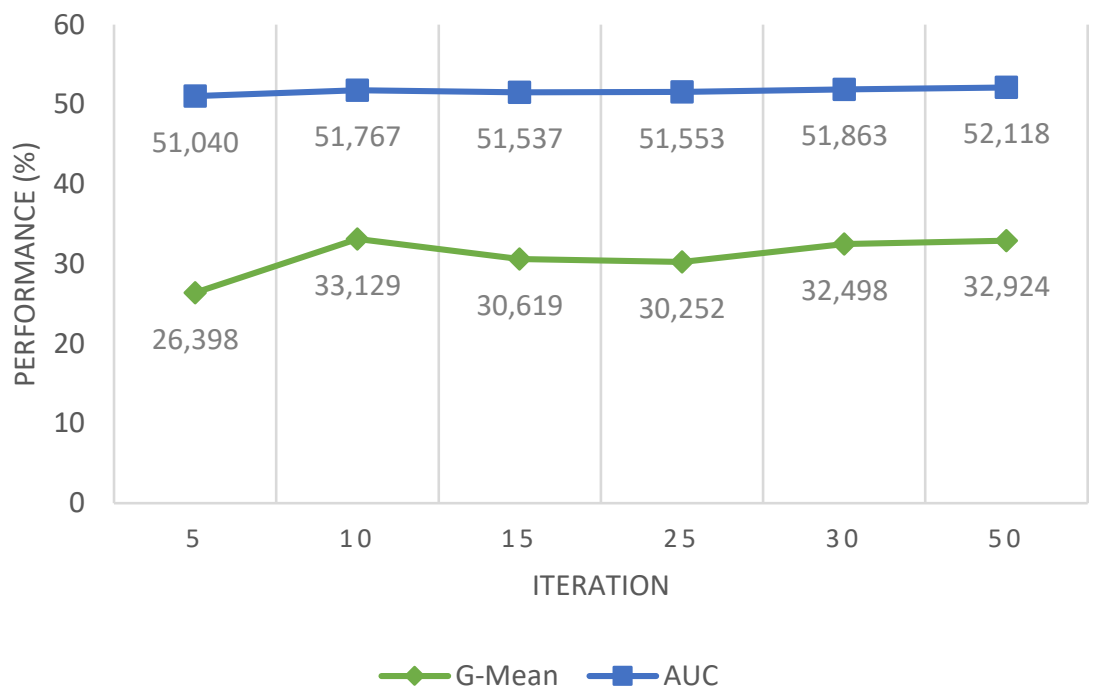

Figure 5. G-mean and AUC Value for SMOTE-Bagging Algorithm 


\section{Comparisson of Performance Classification Methods}

After analyzing the Bidikmisi dataset using AdaBoost, SMOTE-Boosting, and SMOTE-Bagging, the comparison of the method performance of all optimum models is obtained. A comparison of several methods is measured using the performance of classification methods which include precision, recall, f-value, sensitivity, and specificity, g-mean and AUC which are the results of classification with the best parameters of each method. Comparison of the results of the classification is shown in Table 4.4, it can be seen that the performance of all methods shows that SMOTE-Boosting and SMOTE-Bagging have values that tend to be the same. The accuracy of the positive class classification made by the AdaBoost model, which is an average of the fifth fold classification of $8.8235 \%$ which means that on average only $8.8235 \%$ of observations in each fold of the Bidikmisi data have been classified correctly. If seen from the sensitivity and specificity values, AdaBoost can only classify $0.8174 \%$ of observations originating from unaccepted (minority) status as unacceptable classes but succeed in classifying $99.97 \%$ of observations originating from accepted (majority) status as classes received. The existence of imbalance cases in the data causes a low sensitivity value because the random forest separator function tends to classify observations into the majority class, so the classification of minority classes is only correctly classified as less than $1 \%$. After balancing the data in both classes with SMOTE and boosting and bagging, better results were obtained. This is evidenced by the performance of the method with the g-mean value obtained using SMOTE-Boosting random forest and SMOTE-Bagging random forest is higher than AdaBoost.

Table 5. Comparisson of Performance Methods for Bisikmisi Dataset

\begin{tabular}{lcccccccc}
\hline & \multicolumn{1}{c}{ Average 5-fold } \\
\cline { 2 - 9 } \multicolumn{1}{c}{ Model } & Accuracy & Precision & Recall & F-Value & Sensitivity & Specificity & G-mean & AUC \\
\hline AdaBoost & 0,9648 & 0.088235 & 0.008174 & 0.014963 & 0.008174 & 0.999705 & 0.090274 & 0.502561 \\
\hline SMOTE-Boosting & 0,9074 & 0.041885 & 0.100817 & 0.056587 & 0.100817 & 0.937592 & 0.302161 & 0.50757 \\
\hline SMOTE-Bagging & 0,9167 & 0.053549 & 0.119891 & 0.073504 & 0.119891 & 0.947239 & 0.331291 & 0.521178 \\
\hline
\end{tabular}

Based on Table 5. it can also see that AdaBoost produces high accuracy and specificity values. This is because in its boosting process, AdaBoost managed to take advantage of the misclassification made by random forest in each boosting iterations, therefore it can improve the accuracy of classification, especially classification on the majority class. While SMOTE-Boosting and SMOTE-Bagging produce almost the same method of performance values on all criteria due to the process of balancing the distribution of training set classes, resulting in increased classification accuracy in the minority classes. The recall value provides information on how many minority classes are identified but may sacrifice precision by misclassifying the majority class. The F-Value combines precision and recall, to measure the goodness of the learning algorithm.

Fig. 6 presents a boxplot of G-mean values generated in each model. The G-mean value generated using the SMOTE-Bagging shown by the yellow color in the figure tends to be slightly higher compared to SMOTE-Boosting, the value ranges from $26 \%$ to $33 \%$. The blue box is the SMOTE-Boosting, shown that the variation of the G-mean tends to be smaller than the other two algorithms, the value ranges from $26 \%$ to $30 \%$.

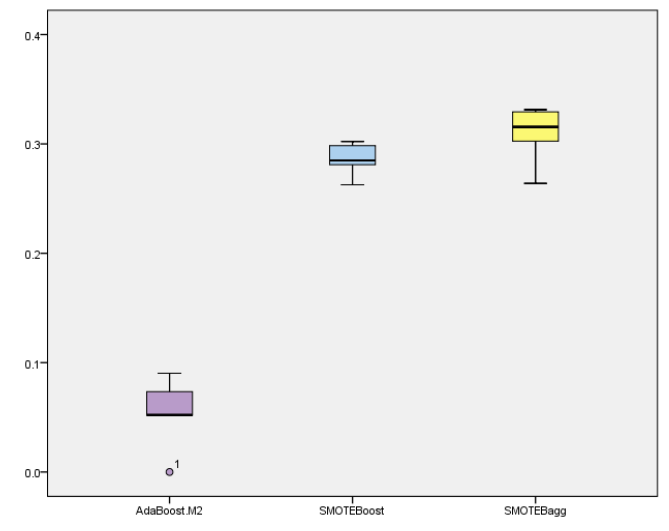

Figure 6. Boxplot Performance of G-mean

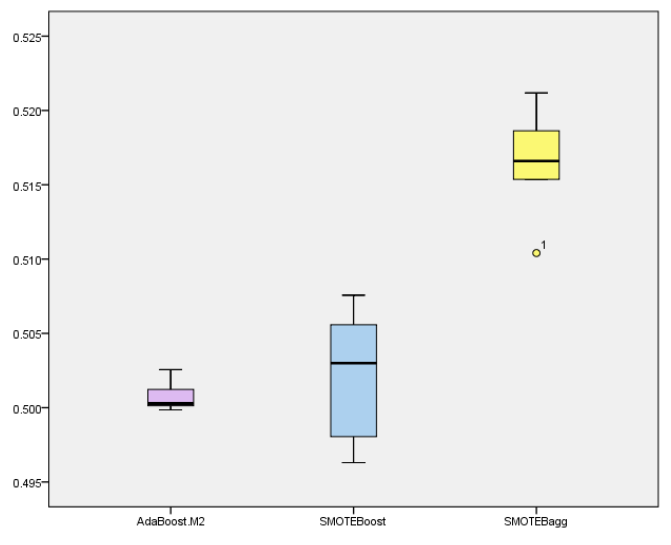

Figure 7. Boxplot Performance of AUC 
Next, Fig. 7 presents a boxplot of the AUC value generated in each model. AUC values generated using the SMOTE-Bagging indicated by the yellow color in the image, tend to be higher than in other methods. The variations range from $51 \%$ to $52 \%$.

\section{Bidikmisi Applicant Classification Results}

Bidikmisi Scholarship is a government scholarship for prospective students who are economically disadvantaged and have good academic potential. Because this scholarship is for poor students, the main requirement for applying for a scholarship is if the income per capita of parents/guardians is divided by the number of family members in the amount of $\mathrm{Rp}$. 750,000.00 every month. Before proceeding to the next discussion, there are several steps undertaken to obtain the values presented in Table 6.

1. Take the variable $\mathrm{Y}$ as the response variable

2. Select variables "father's income", "mother's income", and "family dependant"

3. Create a new variable by counting the amount of "father's income" and "maternal income" divided by "the number of family dependant", name it with "Code category (CC)".

4. Coding the variable "CC" with the following criteria:

$0=$ if CC $>$ Rp. 750,000 per head in the family, included in the condition of a wealthy family

$1=$ if CC $<$ Rp. 750,000 per head in the family, fall into the condition of poor families

5. Match the response variable $(\mathrm{Y})$ to the $\mathrm{CC}$ in Step 4 to the AC (Acceptance Condition) with the Bidikmisi acceptance classification from previous methods (see table 1).

Table 6. Example of The Identification of Bidikmisi Data classification Condition

\begin{tabular}{cccccc}
\hline Object & $\begin{array}{c}\text { Parents Income } \\
\text { (rupiah) }\end{array}$ & $\begin{array}{c}\text { Income per } \\
\text { Capita (rupiah) }\end{array}$ & Actual & Prediction & $\begin{array}{c}\text { Acceptance } \\
\text { Condition }\end{array}$ \\
\hline 1 & 625,000 & 156,250 & 1 & 1 & True \\
\hline 2 & $1,875,000$ & 937,500 & 1 & 0 & False \\
\hline 3 & 875,000 & 875,000 & 1 & 0 & False \\
\hline 4 & $1,375,000$ & $1,375,000$ & 1 & 0 & False \\
\hline 5 & 875,000 & 175,000 & 1 & 1 & True \\
\hline 6 & $1,875,000$ & 625,000 & 1 & 1 & True \\
\hline 7 & $2,750,000$ & 687,500 & 1 & 1 & True \\
\hline 8 & 875,000 & 218,750 & 1 & 1 & True \\
\hline 9 & $1,000,000$ & 500,000 & 1 & 1 & True \\
\hline 10 & $1,250,000$ & 178,571 & 1 & 1 & True \\
\hline
\end{tabular}

Based on Bidikmisi requirements, students are entitled to get a scholarship if the maximum income per capita is Rp. 750,000.00. The results of the "prediction" status of scholarship acceptance indicate that there are 3 students who should not be entitled to get a scholarship, the "actual" results indicate that the ten students turned out to have received scholarship status. In total, there were 10,762 students who get a status "incorrectly classified" condition as shown in Fig. 8.

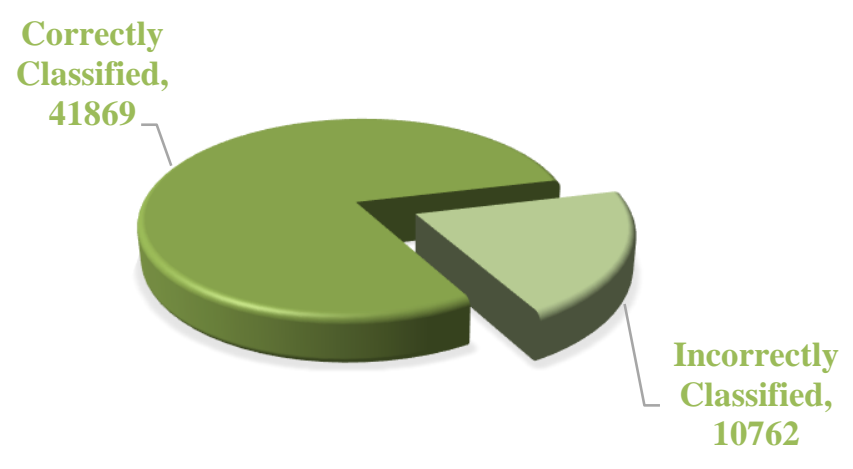

Figure 8. Identification of Bidikmisi Data Classification Condition 
To find out how good the methods used in this research, the prediction results for each method are explained in the following Table 7.

Table 7. Identification of Classification Methods

\begin{tabular}{|c|c|c|c|c|}
\hline Method & Iteration & $\begin{array}{c}\sum \text { Incorrectly } \\
\text { Classified }\end{array}$ & $\begin{array}{l}\sum \text { Correctly } \\
\text { Classified }\end{array}$ & $\begin{array}{l}\text { \% Correctly } \\
\text { Classified }\end{array}$ \\
\hline \multirow{6}{*}{ AdaBoost } & 5 & 356 & 10170 & 96,618 \\
\hline & 10 & 379 & 10147 & 96,399 \\
\hline & 15 & 375 & 10151 & 96,437 \\
\hline & 25 & 376 & 10150 & 96,427 \\
\hline & 30 & 383 & 10143 & 96,361 \\
\hline & 50 & 387 & 10139 & 96,323 \\
\hline \multirow{6}{*}{$\begin{array}{l}\text { SMOTE- } \\
\text { Boosting }\end{array}$} & 5 & 984 & 9542 & 90,652 \\
\hline & 10 & 1083 & 9443 & 89,711 \\
\hline & 15 & 1280 & 9247 & 87,839 \\
\hline & 25 & 1281 & 9246 & 87,830 \\
\hline & 30 & 1259 & 9268 & 88,039 \\
\hline & 50 & 1279 & 9247 & 87,840 \\
\hline \multirow{6}{*}{$\begin{array}{l}\text { SMOTE- } \\
\text { Bagging }\end{array}$} & 5 & 880 & 9464 & 91,639 \\
\hline & 10 & 1192 & 9334 & 88,676 \\
\hline & 15 & 1052 & 9474 & 90,006 \\
\hline & 25 & 1020 & 9506 & 90,309 \\
\hline & 30 & 1123 & 9403 & 89,331 \\
\hline & 50 & 1122 & 9404 & 89,341 \\
\hline
\end{tabular}

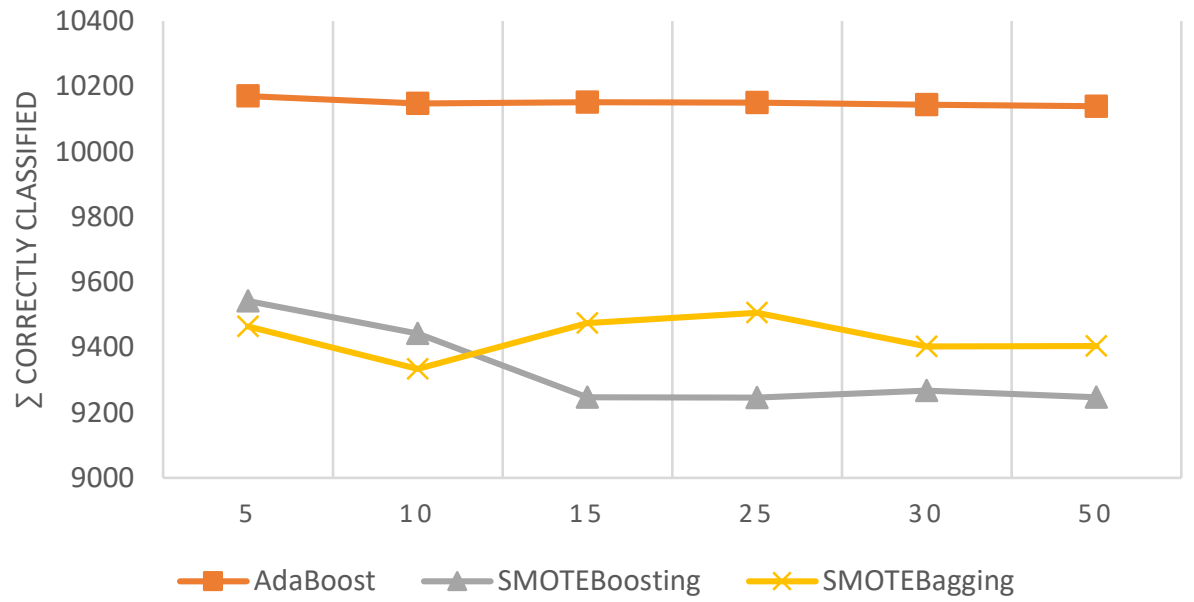

Figure 9. Identification of Classification Methods

\section{Conclusions}

Data mining often used to uncover hidden patterns within a large amount of data. Then, these hidden patterns can be potentially be used to predict future behavior. In relation to Educational Data Mining (EDM) which has been discussed at the beginning, the data mining algorithms were performed to find a prediction for Bidikmisi dataset. It was previously known that the data class has imbalance conditions, therefore, three ensemble algorithms were performed, i.e. Adaptive Boosting, SMOTE-Boosting, and SMOTE-Bagging. All models have been evaluated using stratified 5-fold cross-validation, therefore, the performance criteria for each method are examined.

a. The results of the imbalance class showed that the SMOTE-Bagging and SMOTE-Boosting algorithm has better accuracy than the AdaBoost algorithm. It could be said that both methods were quiete successful in taking advantage the SMOTE algorithm combine with boosting and bagging algorithm. 
b. The predictive value of each method is obtained therefore the accuracy of the classification can be determined by comparing the results of the predictions with the actual value of the classification. The analysis indicates that 10762 applicants were identified as being misclassified. Therefore, this problem needs to be assessed as the target application has not yet been fully achieved.

\section{Acknowledgment}

This research was supported by DPRM-DIKTI under scheme PUPT, project No. 1049/PKS/ITS/2018. The author thanks Kementerian Riset, Teknologi, dan Pendidikan Tinggi for funding and to anonymous references for their useful suggestions.

\section{References}

[1] Baker, Data mining for Education. In McGaw, B., Peterson, P., Baker, E., International Encyclopedia of Education $3^{\text {rd }}$ Edition, Vol.7, Elsevier, United Kingdom, 2010, pp. 112-118.

[2] S.S. Pangastuti, K., Fithriasari, N. Iriawan, W. Suryaningtyas, Classification Boosting in Imbalanced Data. Malaysian Journal of Science, Vol. 8, Special Issue (2) (2019) 36-45.

[3] Directorate General of Learning and Student Affairs, K.R.T.d.P.T. (2018). Bidikmisi Guide 2018, Kemenristek Dikti, Jakarta, 2018.

[4] W. Suryaningtyas, N. Iriawan, K. Fithriasari, B.S.S Ulama, L. Susanto, A.A. Pravitasari, On the Bernoulli Mixture model for Bidikmisi Scholarship Classification with Bayesian MCMC. Journal of Physics: Conference Series, 1090 (2018) 1-8.

[5] R.E. Schapire, Y. Freud, Boosting: Foundations and Algorithms. MIT Press, London, 2018.

[6] L. Breiman, Bagging Predictors. Machine Learning, 1996, 123-140.

[7] N.V. Chawla, K.W Bowyer, L.O Hall, W.P. Kegelmeyer, SMOTE: Synthetic Minority Oversampling technique. Journal of Artificial intelligence research, 16 (2002) 321-357.

[8] S. Wang, X. Yao, Diversity Analysis on Imbalances Data Sets by Using Ensemble Models. IEEE Symp. Comput. Intell. Data Mining (2009) 324-331.

[9] J. Han, M. Kamber, J. Pei, Data Mining Concepts and Technique $2^{\text {nd }}$ Edition. Kaufman Publisher, USA, 2006.

[10] M. Kubat, S. Matwin, Addressing the Curse of Imbalanced Training Sets: One-Sided Selection. Fourteenth International Conference on Machine Learning. Conference Proceedings, (1997) 179-186. 